COMUNICACIÓN CIENTÍfICA IX CONGRESO NACIONAL DE LA SOCIEDAD CIENTÍFICA ESPAÑOLA DE ENFERMERÍA - SCELE. Mayo de 2018. UNIVERSIDAD DE ALICANTE.

\title{
IMPLEMENTACIÓN DE PLANES DE SEGURIDAD DEL PACIENTE: PREVENCIÓN DE CAÍDAS
}

Ruescas-Lopez, Manuel

Chica-Fernández, María José

Torres- Figueiras, María.

Fernández-De-Maya, José.

Gil-Collados, María Carmen.

Soriano-Ruiz, María Josefa.

Hospital Universitario del Vinalopó. Elche. 


\section{PALABRAS CLAVE:}

Pacientes internos, Seguridad del paciente, medición de riesgo, accidentes por caídas.

Introducción: Las caídas son un problema de salud mundial que afecta especialmente a ancianos. Son efectos adversos asociados a la hospitalización, con una presencia del 30 al $50 \%$ en las instituciones sanitarias (1). Siendo parte de los indicadores de seguridad del paciente, la calidad asistencial (2) y la política y estrategia de las instituciones sanitarias españolas (3).

Objetivo: Actualizar el plan de prevención de caídas con los criterios de calidad de la Joint commission on acreditation of Healthcare organizations. Implementarlo en los nuevos soportes informáticos. Describir las cifras de caídas producidas antes y después de la implantación del plan creado.

Metodología: Búsqueda bibliográfica en las principales bases de datos (Pubmed, Cochrane). La selección de resultado se realizó en base a los objetivos alcanzables en cada escenario y se implementó mediante un ciclo de Deming (4). La descripción de números absolutos de caídas antes y después de la implementación del plan.

Resultados: Se realizó la documentación (protocolos, mapas de procesos, evaluación del riesgo, planes de cuidados y registro de caídas) accesible en los soportes informáticos móviles (5), a pie de cama y equipos de las áreas de trabajo $(6,7)$; adaptada a la edad, idioma y área de atención del paciente.

Se ejecutó un plan de difusión y formación sobre la prevención de caídas (On-line y sesiones presenciales). Se formó a los referentes de cada área para desarrollar las políticas de seguridad con sus equipos (8).

Se evaluó la formación on-line mediante test final (8). Se realizaron auditorías internas sobre las principales medidas de seguridad para la prevención de caídas.

El proceso superó auditorías internas y externa de la Joint Commission International. En el año anterior a la implantación se dieron 35 caídas $(0,56 \%$ de caídas por estancia). En el año posterior 33 caídas (0,52\% de caídas por estancia).

\section{Discusión/Conclusión:}

Los protocolos implicados se actualizaron en base a la mejor evidencia disponible. A pesar de las limitaciones de las revisiones sistemáticas y metaanálisis sobre las intervenciones determinantes en la prevención de caídas $(9,10)$, es necesario realizar intervenciones formativas en el personal y familiares, así como actividades evaluación y prevención en la población de riesgo $(11,12)$.

Las herramientas informáticas facilitan el acceso de los mapas de actuación (6), la prevención valoración del riesgo, el seguimiento del plan de prevención, la estandarización de cuidados, de procedimientos y el registro de incidentes $(5,7,13)$.

El diferencial de caídas sufridas antes y después de la implantación del plan es mínimo. Es necesario continuar reevaluando el plan anualmente e implementado nuevas medidas, a la espera de resultados más concluyentes. Es necesario realizar estudios con una serie histórica más amplia para evitar "Cherry picking" en los resultados. 